\title{
Implicaciones del Concepto de Empleabilidad en la Reforma Educativa
}

\author{
Guillermo Campos Ríos \\ Benemérita Universidad Autónoma de Puebla, México
}

\section{Antecedentes}

Dentro de las teorías de los mercados laborales, hay una vertiente importante que enfoca su análisis en las características de la fuerza de trabajo, a la que reconoce la posibilidad de elevar su productividad vía el conocimiento. Esta corriente asevera que la educación es una inversión y que el conocimiento -igual que la salud- radica en los individuos, por lo que se le conoce como la "Teoría del Capital Humano" y es, quizá, la propuesta más influyente dentro del conjunto de teorías que conforman la "Economía de la Educación".

Los principios del capital humano se convirtieron en la teoría usada por los "hacedores de política educativa y laboral" pero al mismo tiempo fue muy cuestionada; en este momento, sigue siendo el horizonte teórico de los diseñadores más prestigiados de las instituciones que actualmente definen los destinos de la educación: el Banco Mundial (BM) y la Organización para la Cooperación y el Desarrollo Económico (OCDE), ambas estructuras de planeación y desarrollo económico de la ONU y que, en la década de los ochenta comenzaron a sustituir a la UNESCO, que era la institución que la propia ONU había creado para el impulso y fomento de la educación.

Más allá de la opinión crítica que sobre esta corriente mantenemos, es obligado referirse a la teoría del capital humano porque todo parece indicar que la noción de empleabilidad es una ampliación y actualización de los principios sostenidos por esta perspectiva teórica, que logra hacerse totalmente evidente en el momento en que la crisis del mercado de trabajo -que ha durado prácticamente tres décadas-. Vuelve a la sobreoferta de fuerza de trabajo en un fenómeno casi permanente en todo el orbe.

El conocimiento es una pieza fundamental del marco explicativo del capital humano, pero no por ello deja de ser un tanto vago, pues lo mismo aglutina al conocimiento formal apropiado en las escuelas, que al conocimiento informal adquirido en fábrica vía la experiencia laboral, o la capacitación, etc. Los tres más destacados autores de la teoría del capital humano: Theodore Shultz, Gary Becker y Jacob Mincer utilizaron el conocimiento como una variable definida fundamentalmente por la escolaridad, sin dejar de reconocer que no es la escuela la única fuente de generación y transmisión de conocimiento..

[...] cuando la gente de origen campesino se ocupa en empleos no agrícolas, ganan sustancialmente menos que los trabajadores industriales de la misma raza, edad y sexo. De manera similar, los hombres no blancos de áreas urbanas ganan mucho menos que los hombres blancos. Estas diferenciales en los ingresos corresponden cercanamente a las diferenciales en educación. Sugieren fuertemente que una es consecuencia de la otra [...] (Shultz: 1961)

Los mismos creadores de la teoría que considera la formación escolar como una inversión señalaron que no se debe identificar al capital humano únicamente con la escolaridad, sino que éste, se haya constituido de un conjunto de atributos entre los que encontramos a las actitudes personales y otros 
rasgos no fácilmente ponderables. Lo que si quedó claro para estos autores fue que el capital humano tiene -en su conjunto- un efecto económico, tanto en los individuos como en las naciones.

Becker concluye que el comportamiento humano se define por aproximaciones económicas que explican a través de instrumentos de mercado las "preferencias" de los individuos y que desde la sociología se imputan a la estructura social. Este mismo autor plantea que en el desempeño laboral, los individuos pueden ser evaluados no sólo por su conocimiento o su escolaridad sino por sus actitudes:

[...]. La productividad de los empleados depende no sólo de su aptitud y de la inversión que se realiza en ellos, tanto dentro como fuera del puesto de trabajo, sino también de su motivación y de la intensidad de su esfuerzo...(Becker: 1983)

La participación cada vez mayor de la OCDE en materia educativa, le ha llevado a la necesidad de difundir una noción actualizada del capital humano, dirigida fundamentalmente a presionar para que los sistemas educativos de los países en desarrollo incluyan dentro de sus programas los nuevos términos de la empleabilidad, aunque en lugar de hablar de actitudes se refiere a competencias. De los documentos de la propia OCDE hemos extraído la siguiente definición de capital humano:

"[...] el capital humano es el conocimiento, las competencias y otros atributos acuerpados en los individuos y que resultan relevantes a la actividad económica [...]" (OECD:1998).

De esa manera, las actitudes laborales son otra manera de identificar un conjunto de maneras de asumir la actividad productiva, pero que a final de cuentas permite orientar cambios tanto del mercado de trabajo como de los sistemas educativos.

Los teóricos del tema en México conciben al capital humano en los siguientes términos: ...(es) el acervo de habilidades, destrezas y conocimiento productivo incorporado en las personas.... (Llamas, 2000)

El capital humano constituye, por tanto, un conjunto de factores tangibles e intangibles, con capacidad de elevar y conservar la productividad, la innovación y la empleabilidad.

En la actualidad, la crisis del mercado laboral con su sobreoferta de fuerza de trabajo y la reducción en las plazas de trabajo han abierto un campo propicio para estimular acciones que incrementan la competencia entre buscadores de empleo y reducen al mínimo el ingreso salarial, promoviendo el estudio de la empleabilidad

En la década de 1990, las transformaciones de los mercados de trabajo fueron destacando a las actitudes; en primer lugar se les transfirió del punto de contratación o "puerto de entrada" al mercado en su conjunto, en segundo lugar se les otorgó un estatus general y no individual y le cambiaron la denominación de "comportamiento laboral" por el de empleabilidad, que es un término más neutro.

También fueron modificándose los criterios de selección de personal, adquiriendo mayor peso las actitudes -y otros factores que no inciden de manera directa sobre la productividad- que los de formación académica. Este cambio no sólo favorece la creación de ambientes laborales más estables, además eterniza relaciones basadas en bajos salarios. Desde este punto de vista, la aplicación y generalización de la noción de empleabilidad sería una de las herramientas idóneas para generar condiciones laborales "flexibles". 
Las nuevas formas de organización de la producción también propiciaron la difusión de las virtudes actitudinales por sobre las de educación, en la misma medida en que caían las hipótesis de "aprendizaje tecnológico" como subproducto del cambio en los patrones de tecnología utilizados.

Fueron los mercados de trabajo de los países más desarrollados los que inicialmente vivieron el cambio en los términos de la oferta y demanda por empleo, de allí que hayan sido también los primeros en dar especificidad a lo que de manera genérica se identifica como actitudes. Los mercados de los países en desarrollo ${ }^{1}$ han ido incorporando a sus esquemas de contratación los componentes de la empleabilidad definidos por los mercados desarrollados, aunque de manera muy particular, pues retoman sólo los aspectos que más les benefician en una visión del mercado laboral de muy corto plazo.

La visión "cortoplacista" de los agentes que conforman los mercados laborales de los países en desarrollo excluye el ya de por si limitado sentido bilateral que en los países desarrollados tuvo el impulso al paquete de exigencias actitudinales.

\section{La Empleabilidad, su Evolución y Significados}

La empleabilidad se entiende como el conjunto de aptitudes y de actitudes que brindan a un individuo la oportunidad de ingresar a un puesto de trabajo y además de permanecer en el.

La palabra empleabilidad no existe como tal en la lengua castellana, proviene de la palabra inglesa "Employability". Esta palabra, a su vez fue construida a partir de "employ", que se traduce como empleo y "hability", cuya traducción es habilidad. Ya unidas formaron: "Employability", la cual se ha adaptado sin mayores cambios al español como "empleabilidad". El significado casi literal que se le otorga a esta palabra es: "habilidad para obtener o conservar un empleo". Una implicación secundaria de esta misma palabra es: la capacidad demostrada en el mercado para evitar el desempleo.

El que la noción de empleabilidad haya surgido en la década de los ochenta aparentemente sin vínculo con la teoría del capital humano y con un marco eminentemente empírico, se explica por un lado por la lentitud de respuesta de la teoría ante la crisis del mercado laboral, pero además porque fue más evidente que para superar los bajos niveles de empleabilidad era necesaria la participación de otros agentes del mercado, como el gobierno y la escuela.

Al inicio, cuando bs mercados laborales no mostraban esta sobreoferta de fuerza de trabajo la empleabilidad era abordada fundamentalmente por los administradores y psicólogos industriales como una fase de la selección de personal, estuvo restringida a un ámbito de acción muy limitado del mercado de trabajo que era el momento de la contratación. A este momento y a este espacio limitado del mercado laboral algunos estudiosos del tema le han llamado el "puerto de entrada". Piore y los segmentalistas lo reconocen como el punto de acceso a los mercados internos (Piore: 1985), una imagen similar manejan Lester Thurow y Keneth Arrow, quienes desde la economía y la administración han explorado el tipo de relaciones que se manifiestan en el momento en que se "enfrentan" físicamente el buscador de empleo y el empleador o el representante de la institución empleadora.

\footnotetext{
Cuando se habla de mercados de trabajo tradicionales no se debe entender un mercado estancado y sin movimiento, sino uno que presenta una menor dinámica de cambio. Los procesos de modernización de los mercados, generalmente están orientados a reducir las brechas que tienen respecto a los mercados desarrollados en el tiempo más breve posible
} 
En el puerto de entrada se harán manifiestas las habilidades de empleabilidad, se confrontarán con la materialización de los nuevos términos de la demanda y con las exigencias que impone el propio puesto de trabajo, el resultado será el éxito o el fracaso en la obtención del empleo. Alrededor de esta confrontación, aparentemente individual, está presionando -lo que algunos modelos conciben como una externalidad económica- el tamaño de la oferta de fuerza de trabajo que existe para ese mismo puesto y que se materializa como una "fila" de aspirantes al mismo puesto de trabajo.

En su formulación más sencilla, la teoría de la fila (o "de la cola", como la identifican Piore y Doeringer) sostiene que los empresarios racionan los puestos existentes entre los trabajadores, de acuerdo con sus preferencias en relación a la contratación. Los trabajadores más solicitados son los primeros de la cola, dejando que los menos solicitados encuentren trabajo en los puestos menos deseables de los sectores marginales de la economía o permanezcan desempleados. Por definición, los desfavorecidos que son los que muestran menor nivel de empleabilidad o hayan realizado menor inversión en capital humano, se encuentran situados al final de la cola de trabajo y tienen un acceso limitado a las oportunidades de empleo más solicitadas. (Piore: 1985).

Desde la perspectiva de la noción de empleabilidad, sólo quedaría por explicar que es lo que define la posición que los buscadores ocupan en la fila; estar al frente o al final de la cola sin duda que no depende de haber llegado antes o después al mercado de trabajo, no es un asunto de temporalidad sino de atributos o habilidades; los que tengan más habilidades de empleabilidad estarán al inicio de la fila.

Algunos segmentalistas más radicales -como Gordon, Edwards y Reich- entenderían que la empleabilidad al manifestarse en el mercado como una fila de espera, es la expresión de que en el proceso de selección de personal juegan factores extraeconómicos como los políticos, los raciales, etc. Y para ellos, la empleabilidad estaría ocultando factores de decisión que no siendo económicos forman parte del funcionamiento de los mercados, son parte de su institucionalidad y sería de esperar la presencia de aspirantes que estando al final de la cola se puedan eternizar en una espera inútil de un puesto de trabajo que nunca llegará.

Lester $\mathrm{C}$. Thurow sostiene que el entrenamiento y la educación no son factores importantes para determinar la productividad potencial de los trabajadores, dado que la productividad es un atributo de los puestos de trabajo y no de la mano de obra (THUROW: 1972),. Los empleos asociados con equipos de capital y tecnologías modernas son empleos de alta productividad, y los trabajadores "hacen fila" a la espera de un empleo en ese sector. Una vez que el trabajador es contratado, las destrezas cognitivas necesarias para elevar su productividad al nivel de la productividad del puesto son aprendidas a través de programas de entrenamiento que pueden ser formales o informales.

De esta manera, según Thurow, el criterio que los empleadores usan al seleccionar a los trabajadores, es la entrenabilidad; aquellos que poseen antecedentes y características que los empleadores piensen pueden reducir los costos del entrenamiento van a la cabeza de la fila y reciben -pronto- el mejor trabajo.

De manera similar, Kenneth Arrow, sugirió que la educación puede ser un mecanismo para distinguir a los trabajadores deseables de bs no deseables. Esta hipótesis también recibe el nombre de teoría del "filtro" (ARROW: 1972), que al igual que el efecto de fila, implican que la educación no contribuye directamente al crecimiento de la productividad de los individuos, pero sirve como un medio para clasificar a 
las personas según los certificados escolares que hayan obtenido y que pueden dar muestra de una mayor disciplina, tenacidad, responsabilidad, etc.

En la visión de Thurow y Arrow, el puerto de entrada ayudaría a resaltar lo que es importante en la definición de la empleabilidad, disminuyendo la importancia de la formación escolar y destacando la importancia de las actitudes, pero igualmente, encontramos una percepción individualizada de los rasgos que distinguen a cada trabajador.

Con la sobreoferta de fuerza de trabajo en los mercados laborales la empleabilidad se planteó como un problema no individual, sino general, era la nueva "norma" no escrita que brinda mayor grado de libertad al empleador para seleccionar a su personal; fue la evidencia de que las reglas de juego de este mercado estaban cambiando, favoreciendo aún más al empleador y, si bien el mercado de trabajo siempre había sido dominado por el empleador, había una mínima regulación; ahora, con la noción de empleabilidad se "mina" la posibilidad de una relación laboral menos desigual, limitando la normatividad que anteriormente protegía de manera relativa a la fuerza de trabajo -ocupada- a través de sus organizaciones de representación como los sindicatos

En diversos reportes se expresa que la insatisfacción de los empleadores con los buscadores de empleo jóvenes no se debe en principio a un conocimiento técnico inadecuado:

"...una revisión de la literatura indica que los empleadores no tienen problema con el comportamiento de las habilidades técnicas de los graduados actuales, ellos tienen graves reservas en cuanto a las habilidades no técnicas" (Wentling: 1987).

Kateleen Cotton realizó una investigación sobre los criterios más ampliamente difundidos entre los empresarios para identificar y elegir a su personal en función de sus atributos de empleabilidad, lo encontrado por esta investigadora se puede resumir en un conjunto de nuevas exigencias que engloban por un lado competencias básicas que permiten la comunicación y, por đro, actitudes y buena disposición al trabajo en equipo. Para su presentación las organizó en tres categorías de demanda: habilidades básicas; habilidades de pensamiento de alto nivel y habilidades afectivas y de trato (Cotton: 1993). Es notorio el predominio de las habilidades relacionadas con la afectividad y la capacidad de ofrecer un buen trato no sólo a los subordinados, sino a los compañeros de trabajo. Todas aquellas denominadas afectivas, particularmente la "formalidad", "responsabilidad" y "actitud positiva hacia el trabajo" son vitales desde el punto de vista de los empleadores.

Los resultados confirman que las habilidades ocupacionales específicas son menos cruciales para los empleos (especialmente los de primera ocasión) que un nivel general de habilidades de lectura, de responsabilidad, de actitudes hacia el trabajo, la habilidad de comunicarse bien y la habilidad para continuar aprendiendo. Beach cita investigaciones que indican que: ...el $87 \%$ de las personas que perdieron sus empleos o no fueron promovidos se debió a que tenían "inapropiados hábitos de trabajo y mala actitud más que falta de conocimiento o habilidades de trabajo"... (Beach: 1982).

De estas investigaciones es que se fue desprendiendo la idea de que la empleabilidad es la evidencia empírica de que los mercados están cambiando, orientándose a la disminución del riesgo productivo a través de construir ambientes de "máxima estabilidad". 
Sin embargo, el costo de los nuevos ambientes laborales corre a cargo de los buscadores de empleo, los cuales deberán llegar con las nuevas habilidades ya desarrolladas y convencidos de la necesidad de compartir intereses con la empresa. Estas habilidades las deberá desarrollar de manera individual, aunque recientemente se ha comenzado a reconocer su importancia social, al punto que también se plantea como una responsabilidad de la escuela y de los gobiernos.

\section{La Empleabilidad y el Nuevo Papel de la Escuela.}

En ciertos momentos, la formación de los recursos humanos se consideró una labor compartida, algunas empresas llegaron a constituir verdaderas escuelas de capacitación para su personal; el gobierno promovió la creación de institutos de capacitación para el trabajo y de recualificación de competencias, la escuela por su parte generó alternativas específicas de formación para el trabajo y adaptó modelos de formación propedéutica y para el trabajo. Sin embargo, los resultados nunca llegaron a cubrir las expectativas originalmente concebidas.

Esta visión de la formación de recursos humanos como una labor compartida aún subsiste pero con severas limitaciones, especialmente en lo que toca a las responsabilidades que corresponden al sector empresarial, el cual a través de sus voceros permanentemente expresa que la capacitación implica un costo doble, ya que por un lado pagan impuestos que se canalizan a una escuela que debiera dar esta capacitación y, por otro, deben gastar en capacitación del personal de nueva contratación y en recualificar al personal ya contratado.

El papel que juega la noción de empleabilidad en esta polémica es de plantear que dichas habilidades son una responsabilidad individual, especialmente de aquellas que se desarrollan de manera preferente en los hogares y en los ambientes contextuales más inmediatos al hogar. Con esta propuesta se establece paralelamente una suerte de desajuste entre las necesidades de los individuos y los contenidos educativos de la escuela, haciendo aparecer a ésta como totalmente desfasada de sus usuarios.

Ante el panorama de cambios que por su parte enfrenta la escuela, un elemento clave de su orientación es la necesidad de satisfacer las necesidades de formación de sus estudiantes, determinadas en mucho por las expectativas que tendrán en el mercado de trabajo y éste envía señales muy claras sobre las habilidades de empleabilidad que está solicitando el mercado. Este razonamiento se puede volver circular con mucha facilidad e impedir llegar a la mejor solución sobre el cambio más adecuado para la escuela.

Los empleadores estiman que las escuelas deben asumir su responsabilidad y otorgar el máximo de habilidades básicas a los jóvenes que forman."...Los maestros de educación industrial en las escuelas secundarias no deberían olvidar que hay una gran necesidad para preparar a la gente joven en sus respectivas clases con buenos hábitos de trabajo. Los estudiantes necesitan pensar en cosas como la honestidad, la puntualidad, la atención regular, la productividad y la rigurosidad..." (Lundy: 1984) 
“...El mundo ha cambiado", escriben los autores del reporte SCANS de 1991 haciendo referencia a los días cuando "una espalda fuerte, el simple deseo de trabajar y un diploma de secundaria era todo lo que se necesitaba para empezar... En lugar del trabajo rutinizado, repetitivo y organizado a lo largo de una serie jerárquica, el mercado de trabajo moderno requiere diferentes tipos de tareas, enfoques y empleados.

En el otro extremo están quienes reconocen que es indudable que la escuela debe cambiar, pero aún así, pueden identificar objetivos fundacionales que sostienen su validez y permanencia. La escuela no puede convertirse en un vasallo silencioso de la estructura productiva. Por tanto no puede abandonar ciertos objetivos de formación para satisfacer única y exclusivamente las necesidades del mercado.

La escuela contribuye a la formación de recursos humanos y en ese proceso influyen múltiples eventos académicos y de relación intelectual, queda pendiente el análisis del efecto que puede dejar la noción de empleabilidad sobre los cambios escolares, especialmente si se considera que las habilidades de empleabilidad no son susceptibles de adquirirse a través de un mero entrenamiento, sino que implica cambios importantes en la forma en que se desempeñaría la escuela.

Desde el ámbito escolar, las habilidades de empleabilidad se pueden identificar con el desarrollo de competencias básicas de empleo, en ese sentido, podría ser interesante incluir la discusión sobre la empleabilidad en la definición de las competencias porque permitiría centrar la discusión -sobre cómo lograrlas - en puntos que implican un cambio fuerte en las relaciones y formas de ser de la escuela actual y no sólo verlas como un conjunto de nuevas materias (entrenamientos) que se imponen mecánicamente.

En este momento existe una estrategia promovida por el Banco Mundial para que el nivel medio superior en México transforme sus contenidos con base en un modelo basado en competencias laborales, el cual coincide con las demandas del sector productivo por incrementar las habilidades de empleabilidad. En general, este modelo se aplicará de manera mecánica y al final es previsible que poco contribuirá a un cambio real de la formación de recursos en este nivel. Aquí sería importante involucrar a los docentes y autoridades del nivel medio superior en una discusión sobre las articulaciones entre competencias laborales y habilidades de empleabilidad, así como los cambios que hay que realizar para lograrlas.

En el mismo orden de ideas, tenemos que el nivel superior en México lleva varios años en el camino de ajustar sus programas de estudio hacia el logro de mayores habilidades de competencia, hacia el logro de la certificación de los estudios universitarios. El saldo es una fuerte tensión entre quienes están dispuestos a eliminar contenidos que no influyen directamente sobre la empleabilidad y otros que defienden la necesidad de conservar elementos de formación más integral.

Los sistemas educativos son cada vez más parecidos entre si, les ocurre algo similar que a los mercados, los sistemas educativos más desarrollados van presionando a los menos desarrollados a que acepten modificaciones que hayan sido exitosas. Es un hecho que en diversos sistemas educativos han proliferado los modelos de aprendizaje basados en competencias y de la misma manera se irá volviendo más común en México la discusión de los cambios hacia modelos de competencias. Por su parte el mercado de trabajo estará presionando a los buscadores de empleo a que dispongan de un mayor número de habilidades de empleabilidad. Pareciera el momento de iniciar un proceso de discusión más profundo sobre las implicaciones entre estos dos términos y analizar sin prejuicio los beneficios que se pudieran derivar de su aplicación hacia los modelos de educación que irá asumiendo en el corto plazo la escuela mexicana. 
Finalmente, la noción de empleabilidad nos lleva a reconocer que el mercado de trabajo es una institución, que ésta se mantiene en permanente cambio y que en este momento de crisis se inclina aún más a favor de los empleadores. En este marco de relaciones desiguales, se ha relativizado la importancia que tradicionalmente ha tenido la educación en la definición del empleo y el salario y ha adquirido una gran relevancia un paquete de actitudes que pueden conformar ambientes de mayor productividad, sin embargo la conformación de estos ambientes corre a cargo de los buscadores de empleo.

La empleabilidad también ayuda a recordar que en el proceso de cambio del mercado también sus agentes principales cambian y, en este caso, restringiéndonos sólo a la escuela que a su vez tiene su propia dinámica de cambio, debe considerar más reflexivamente el efecto de las nuevas habilidades de empleabilidad en la definición de las transformaciones que está realizando a sus modelos y no adaptar mecánicamente algún caso exitoso que se haya dado en otro país.

\section{Referencias Bibliográficas}

ARROW, Keneth. Higher education as a filter. Technological report. Universidad de Stanford. USA. (1972).

BECKER, Gary. Inversión en capital humano e ingresos, en Luis de Tohaira (compilador) El mercado de trabajo: teorías y aplicaciones. Alianza Universidad. España. (1983).

BECKER, Gary. The Foundations of Human Behavior. En The essence of Becher . Ed. Hoover Institution Press. USA. (1995).

BRAUDEL, Fernand. Civilización material, economía y capitalismo, siglos XV-XVIII. Tomo II, Los juegos del intercambio. Ed. Alianza Editorial. España. (1979).

COTTON, Kathleen. Developing Employability Skills. School Improvement Research Series. (SIRS) CloseUp \#15. (1993).

LLAMAS H. I. Educación y Mercado de Trabajo en México. Ed. UAM-I. México. (1989).

PIERSON, F. An evaluation of Wage Theory. En George W. Taylor et al "New concepts in wage determination". Ed. Mc. Graw Hill. EUA (1957)..

Shultz, T. Investment in human capital. American economic review, [Vol. 51 pp. 1-17]. USA. (1961).

PIORE, Michel. y DOERINGER, Peter. (1985). Mercados internos de trabajo y análisis laboral. $2^{\circ}$ edición. Ministerio de Trabajo y Seguridad Social. España

SATERFIEL, Thomas H. - MCLARTY, Joyce R. Assessing Employability Skills. ERIC Digest. Greensboro NC. (1995).

SMITH, Jeremy, MCKNIGHT Abigail and NAYLOR Robin "Graduate Employability: Policy and Performance in Higher Education in the UK'. Economic Journal (junio). UK. (2000).

STRAWN, Julie. Beyond Job Search or Basic Education: Rethinking the Role of Skills in Welfare Reform. CLASP. (1998).

THUROW, L y LUCAS, R. The american distribution of income: a structural problem. Washington. USA. (1972). 
THUROW, L. El Futuro del Capitalismo. Ed. Javier Vergara. Buenos Aires. (1996). 